des Côtes d'Europe. Mm. Soc. Natn. Sci. Nat. Math.. Cherbourg 41: 1-236.

GEITLER, L. - 932- Cyanophyceae, In : Rabenhorst's Kryptogamen flora. Akademische Verlagsgesellschaft Lepzig. 1196 pp.

NAGARKAR, S. -1998- New records of marine cyanobacteria from Rocky shores of Hong Kong. Bot. Mar. 41:527-542.

SANDGREEN, C. D. -1988- Growth and reproductive strategies of freshwater phytoplankton. Cambridge University Press, New York.

STARMACH, K. -1966- Cyanophyta - Sinice, Glaucophyta - Glaukofity, Flora slodkowodna Polski, tom 2, Warszawa. 800 pp.
THAJUDDIN, N. and SUBRAMANIAN, G. -1992Survey of cyanobacterial flora of the southern east coast of India. Bot. Mar. 35 : 305- 14.

Aceptado para su publicación en abril de 2000

Author's address: Post Graduate Department of Biosciences. Sardar Patel University. Vallabh Vidyanagar 388 120. Gujarat. India . Fax: + 912692 36475.

\title{
86. NUEVAS ADICIONES AL CONOCIMIENTO DE LAS MACROALGAS MARINAS DE LA ISLA DE ALBORÁN (MEDITERRÁNEO OCCIDENTAL)
}

\author{
Francisco CONDE y Antonio FLORES-MOYA
}

Contribution to the knowledge of the seaweeds of Alboran Island (Western Mediterranean).

Palabras clave. Algas marinas, Dictyosphaeria ocellata, Gastroclonium ovatum, Isla de Alborán

Key words. Alboran Island, Dictyosphaeria ocellata, Gastroclonium ovatum, Seaweeds

La composición específica de la flora de macroalgas marinas de la isla de Alborán es conocida a través de los trabajos de SotoMoreno y Conde-Poyales (1993) y Rindi \& Cinelli (1995), así como por las contribuciones sobre los géneros Phyllariopsis (Izquierdo et al., 1995) y Predaea (Conde et al., 1998). A partir de estos trabajos se conocen un total de 160 taxones específicos e infraespecíficos de macroalgas marinas de Alborán.

Con el objeto de aumentar el conocimiento sobre la flora de macroalgas marinas de Alborán, se han identificado las muestras recogidas en cuatro campañas, desde 1994 a 1996: 1) abril de 1994 (Departamento de Biología Vegetal de la Universidad de Málaga); 2) septiembre de 1994 (Instituto Español de Oceanografía, IEO); 3) junio y julio de 1995 (IEO); 4) julio de 1996 (Proyecto Fauna IV, Museo Nacional de Ciencias Naturales, Madrid). Todas las muestras se recogieron desde la zona litoral hasta alrededor de $100 \mathrm{~m}$ 
profundidad; para ello se emplearon diversas técnicas: recogida a mano, tanto en superficie como con escafandra autónoma hasta $60 \mathrm{~m}$ de profundidad, y mediante dragas de arrastre de bou de varas hasta donde desaparecía la flora. El material se fijó con agua de mar formolada al 4\%. Con la metodología usual se identificó posteriormente todo el material, y fue prensado y depositado en el herbario del Departamento de Biología Vegetal, Facultad de Ciencias, Universidad de Málaga (MGC-Phyc).

De los 110 taxones encontrados, 59 se han citado anteriormente y, junto con las 51 nuevas citas (8 Fucophyceae, 9 Chlorophyceae y 34 Rhodophyceae), dan lugar a un catálogo de 211 taxones para Alborán (42 Fucophyceae, 27 Chlorophyceae y 142 Rhodophyceae).

De las nuevas citas cabe destacar a Dictyospheria ocellata (Howe) OlsenStäjkovich y Gastroclonium ovatum (Hudson) Papenfuss, especies no citadas hasta ahora para el Mediterráneo.

La flora de Alborán tiene un índice R/P (Rhodophyceae/Fucophyceae) (Feldmann 1938) y $(\mathrm{R}+\mathrm{C}) / \mathrm{P}$ (Rhodophyceae + Chlorophyceae) / Fucophyceae (Cheney 1977) de 3.38 y 4.02 , respectivamente. El valor del índice $\mathrm{R} / \mathrm{P}$ es similar a lo de las costas mediterráneas de la Península Ibérica y Andalucía (Gallardo et al., 1985, Flores-Moya et al., 1995a, 1995b, Conde et al., 1996).

Para la ordenación de la lista de taxones se han seguido los criterios de clasificación de la check-list de Andalucía (Flores-Moya et al., 1995a, 1995b, Conde et al., 1996).

\section{FUCOPHYCEAE Warmyn}

Nemoderma tingitanum Schousboe ex Bornet Sporochnus pedunculatus (Hudson) C. Agardh Arthrocladia villosa (Hudson) Duby Colpomenia peregrina (Sauvageau) Hamel

Dictyota dichotoma (Hudson) Lamouroux var. intricata (C. Agardh) Greville

Dilophus fasciola (Roth) Howe
Cystoseira brachycarpa J. Agardh var. balearica

(Sauvageau) Giaccone (=C. balearica Sauvageau)

C. sauvageauana Hamel

\section{CHLOROPHYCEAE Wille s. 1.}

Blidingia marginata (J. Agardh) P. Dangeard (=Enteromorpha marginata J. Agardh)

B. minima (Nageli ex Kützing) Kylin

Cladophora coelothrix Kützing

C. pellucida (Hudson) Kützing

C. retroflexa (Bonnemaison ex Crouan frat.) van den Hoek

Dictyosphaeria ocellata (Howe) Olsen-Stäjkowich Codium adhaerens $\mathrm{C}$. Agardh

C. bursa (L.) C. Agardh

C. fragile (Sur) Hariot var. tomentosoides (Van Goor) Silva

\section{RHODOPHYCEAE Rabenhorst}

Bonnemaisonia asparagoides (Woodward) C. Agardh

Callithamnion corymbosum (Smith) Lyngbye

Ceramium ciliatum (J. Ellis) Ducluzeau

Crouania attenuata (C. Agardh) J. Agardh f. bispora (Crouan) Hauck

Wrangelia penicillata $\mathrm{C}$. Agardh

Nitophyllum punctatum (Stackhouse) Greville

Chondria dasyphylla (Woodward) C. Agardh

Laurencia obtusa (Hudson) Lamouroux

Polysiphonia brodiaei (Dillwyn) Sprengel (=Hutchinsia brodiaei C. Agardh)

P. fibrillosa (Dillwyn) Sprengel

P. pulvinata C.Agardh

Pterosiphonia ardreana Maggs et Homersand

P. complanata (Clemente) Falkenberg

P. pennata (C. Agardh) Falkenberg (=Polysiphonia pennata (Roth) Falkenberg)

Amphiroa beauvoisii Lamouroux

Lithothamnion coralloides Crouan

Phymatolithon calcareum (Pallas) W. Adey et McKibbin

P. lenormandii (J. Areschoug) W. Adey

Gelidium pectinatum Schousboe ex Montagne

Gelidiella nigrescens (J. Feldmann) J. Feldmann et Hamel

Calliblepharis ciliata (Hudson) Kützing

Grateloupia filicina (Lamouroux) C. Agardh 
Hypnea musciformis (Wulfen) Lamouroux Kallymenia microphylla J. Agardh

K. reniformis (Turner) J. Agardh

Peyssonnelia rubra (Greville) J. Agardh

P. stoechas Boudouresque et Denizot

Gymnogongrus crenulatus (Turner) J. Agardh

G. griffithsiae (Turner) G. Martius

Gracilaria multipartita (Clemente) Harvey

G. verrucosa (Hudson) Papenfuss

Scinaia turgida Chemin

Chylocladia verticillata (Lightfoot) Bliding

Gastroclonium ovatum (Hudson) Papenfuss

Sporochnus pedunculatus y Arthrocladia villosa son especies atlántico-boreales del infralitoral y circalitoral. En la Isla de Alborán se han encontrado a $-34 \mathrm{~m}$ y $-37 \mathrm{~m}$, en fondos con fuertes corrientes, caracterizados por la presencia de Laminaria ochroleuca, Desmarestia dresnayi, Phyllariopsis brevipes, P. purpurascens, Cystoseira usneoides, rodolitos, Phymatolithon calcareum, $P$. lenormandii, Calliblepharis ciliata y Kallymenia sp pl.

Cystoseira brachycarpa var. balearica y C. sauvageauana son especies de distribución mediterránea, casi restringidas al Mediterráneo Occidental, aunque ambas se han encontrado en el litoral atlántico de Cádiz (Gómez-Garreta etal., 1992; Flores-Moya et al., 1995a). Ambos taxones se hallaron en Alborán a poca profundidad, en una comunidad de algas fotófilas junto con el erizo de mar (Paracentrotus lividus) y Lithophyllum incrustans.

La presencia de Bonnemaisonia asparagoides, de distribución atlántico-boreal y mediterránea, presente en fondos de laminarias, próxima a los $-40 \mathrm{~m}$, caracteriza la entrada de las aguas frías del Atlántico; este taxon también se conoce para las costas de Almería y Cádiz (Conde et al., 1996).

Amphiroa beauvoisii es una especie indoatlántica tropical, cuyo límite septentrional sea, posiblemente, el sur de Portugal (Ardré, 1970); este taxon está ampliamente distribuido por Andalucía y todo el Mediterráneo Occidental. Es relevante su presencia en la Isla de Alborán, en tanto que supuestamente su introducción ha sido a través del Estrecho de Gibraltar para su posterior dispersión por el Mediterráneo.

Codium fragile var. tomentosoides y Colpomenia peregrina son dos nuevas citas para la Isla de Alborán, provenientes del Pacífico y, como tal, invasoras que han penetrado por el Estrecho de Gibraltar difundiéndose por el Mediterráneo Occidental. La primera es un elemento ficogeográfico Atlántico-Pacífico templado-frío, mientras que C. peregrina está considerada CircumboreoAustral, con distribución relativamente amplia en Andalucía. Sin embargo, $C$. fragile var. tomentosoides no se ha citado para Andalucía (Flores-Moya et al., 1995b), pero hay que señalar que al revisar las muestras del herbario MGC, se encuentran ejemplares de las provincias de Cádiz, Málaga y Granada.

Maggs \& Hommersand (1993) propusieron Pterosiphonia ardreana para recoger a $P$. spinifera (Kützing) Falkenberg var. robusta Ardré. Ardré (1970) comentó que la variedad difiere del tipo por su aspecto más robusto. Miranda (1931: 65) señaló la existencia en las costas cantábricas españolas de una $P$. pennata (Roth) Falk. muy robusta, cuyo aspecto recuerda a $P$. dendroidea Kütz. Por todo ello Ardré (1970) comenta que los ejemplares de Miranda deben ser pasados al taxon expuesto. Aunque el perfil del conjunto parece más triangular (cf. $P$. parasitica) que linear, el hecho de no presentar los ejemplares de Alborán ejes postrados y ser la anchura del talo menor de $10 \mathrm{~mm}$ (aproximadamente 4 $\mathrm{mm}$ ), además de la anchura de los ejes mayores (entre $400 \mu \mathrm{m}$ y $500 \mu \mathrm{m}$ ), llevan a la conclusión de que el taxon de Alborán es $P$. ardreana. La distribución de esta especie parece ser, además del Mediterráneo, desde las Islas Británicas a Portugal. El material procede de un arrastre entre -29 m y $-35 \mathrm{~m}$; la flora acompañante 
estaba compuesta por individuos de Saccorhiza polyschides, Phyllariopsis brevipes, $P$. purpurascens, Laminaria ochroleuca, Zonaria tournefortii, Calliblepharis ciliata y Asparagopsis armata.

Gastroclonium ovatum es una especie que se distribuye por el noreste atlántico, desde el Reino Unido a Mauritania. Posiblemente la existencia de este taxon en la Isla de Alborán a -16 m sea debida a la penetración de las aguas atlánticas. Esta es, probablemente, la primera cita de esta especie para el Mediterráneo.

Dictyosphaeria ocellata es una especie pantropical, encontrada como epífita de Cystoseira tamariscifolia. Se considera también nueva cita para el Mediterráneo. Esta especie se ha identificado como tal por la presencia en su base de largas células rizoidales (Taylor, 1960).

Se resaltan, como segundas citas, a Apoglossumm ruscifolium, Ceramium echionotum, Dasya hutchinsiae, Desmarestia dresnayi, Gracilaria bursa-pastoris y Plocamium cartilagineum.

Aún faltando por inventariar desde los meses de octubre a marzo (según se deduce de los trabajos previos y esta contribución), y habiendo sido el muestreo suficientemente extenso en batimetría (desde el nivel cero hasta los -60 m con escafandra autónoma, y hasta los - $100 \mathrm{~m}$ con arrastre de bou de varas), se puede concluir que con las nuevas aportaciones el presente catálogo sobrepasa los 200 taxones.

Conde Poyales (1989) propuso que la Isla de Alborán se encontraba en una una zona límite o intermedia entre los sectores atlánticos y mediterráneos. Esta idea se ve confirmada por la inexistencia de: i) endemismos mediterráneos como Rissoella verruculosa y Posidonia oceanica; ii) Fucales atlánticos templado-frios de los géneros Bifurcaria, Halidrys, Ascophyllum, Fucus, Pelvetia e Himanthalia; y iii) clorofíceas pan- o tropicales de los géneros Acetabularia, Anadyomene, Dasycladus y Halimeda. Por otra parte, la presencia de las especies atlánticos-boreales Laminaria ochroleuca (grandes bosques casi homogéneos entre -25 m y -40 m), Saccorhiza polyschides, Phyllariopsis brevipes, $P$. purpurascens y Cystoseira sp pl., incluso algunas mediterráneas, no hacen sino apoyar la hipotesis en la que la semejanza es mayor con las costas más occidentales de la provincia de Málaga, pero sin llegar a las de Cádiz, y otro tanto pasaría con relación a las occidentales de Marruecos (González García \& Conde Poyales, 1994).

AGRADECIMIENTOS. Deseamos agradecer el que nos dejaran participar a uno de nosotros (AFM) en algunas de estas campañas; también a todas aquellas personas que nos recogieron muestras de forma desinteresada.

\section{BIBLIOGRAFÍA.}

ARDRÉ, F. -1970- Contribution à l'étude des algues marines du Portugal. Portugaliae Acta Biologica (Série B), 10: 137-532.

CHENEY, D.P. -1977- A new and improved ratio for comparing seaweed floras. J. Phycol., 13 (suppl.): 13.

CONDE POYALES, F. -1989- Ficogeografía del Mar de Alborán en el contexto del Mediterráneo Occidental. An. Jard. Bot. Madrid 46(1): 21-26. CONDE F., A. FLORES MOYA, J. SOTO, M ALTAMIRANO y A. SANCHEZ -1996- Checlist of Andalusia (S. Spain) Seaweeds. III. Rhodophyceae. Acta Bot. Malacitana, 21: 7-33.

CONDE, F., C. LOPEZ MIELGO y A. FLORES MOYA -1998- The genus Predaea (Nemastomatacea, Rhodophyta) in the Alborán Sea (western Mediterranean Sea), with the description of Predaea pusilla f. alboranensis f. nov. Phycologia 37(5): 394-397.

FELDMANN, J. -1938- Recherches sur la Végétation Marine de la Méditerranée. La Côte des Albères. Rev. Algol., 10(1-4): 1-339.

FLORES MOYA A., J. SOTO, A. SANCHEZ, M. 
ALTAMIRANO, G. REYES y F. CONDE 1995a- Check-list of Andalucía (S. Spain) seaweeds. I. Phaeophyceae. Acta Bot. Malacitana, 20: 5-18.

FLORES MOYA, A. J. SOTO, A. SANCHEZ, M. ALTAMIRANO, G. REYES y F.CONDE 1995b- Check-list of Andalucía (S. Spain) seaweeds. II. Chlorophyceae. Acta Bot. Malacitana, 20: 19-26.

GAllardo, T., A. GomeZ GARRETA, M. A. RIBERA, M. ALVAREZ y F.CONDE - 1985- A preliminary checklist of Iberian benthic marine algae. Real Jardín Botánico. Madrid. 83 pp.

GÓMEZ GARRETA, A., M. C. BARCELO, J. RULLLLUCH, M. A. RIBERA y J. ECHEGARAY. -1992Apports chorologiques du Genre Cystoseira C. Agardh. Rapp. Comm. int. Mer Médit., 33: 40.

GONZALEZ GARCIA, J. A. y F. CONDE POYALES 1994-Catálogo del macrofitobentos del Mediterráneo de Marruecos. Acta Bot. Malacitana, 19: 5-27.

IZQUIERDO, J. L., F. CONDE y A. FLORES MOYA -1995-Adiciones a la distribución geográfica del género Phyllariopsis Henry et South (Laminariales Phaeophyta) en la Península Ibérica e Islas Baleares. Acta Bot. Malacitana, 20:281-283.
MAGGS C. A. \& HOMMERSAND M. H. -1993Seaweeds of the British Isles. Vol. 1 . Rhodophyta. Part 3A. Ceramiales. British Museum: HMSO. London. 444 pp.

MIRANDA F. - 1931- Sobre las algas y Cianofíceas del Cantábrico, especialmente de Gijón. Trab. mus. Nac. Ci. Nat., Ser. Bot., 25: 1-106.

RINDI, F. \& F. CINELLI -1995-Contribution to the knowledge of the benthic algal flora of the Isle of Alboran, with notes on some little-know species in the mediterranean. Cryptogamie, Algol., 16(2): 103-114.

SOTO MORENO, J. y F. CONDE POYALES -1993Datos sobre la flora algal bentónica de la Isla de Alborán (Mar de Alborán. Mediterráneo Occidental). Cryptogamie, Algol., 14(4): 183-190.

TAYLOR, R. -1960- Marine algae of the eastern tropical and subtropical coasts of the Americas. The university of Michigan Press. 870pp.

Aceptado para su publicación en julio de 2000

Dirección de los autores. Departamento de Biología Vegetal. Facultad de Ciencias Universidad de Málaga. Apdo. 59. 29080 Málaga.

\title{
87. BRIÓFITOS NUEVOS O INTERESANTES PARA LA FLORA DE GALICIA
}

\author{
Juan REINOSO FRANCO y Aida GARCÍA MOLARES
}

Bryophytes new or rare for the flora of Galicia

Palabras clave. Briófitos, corología, flora, Galicia, España.

Key words. Bryophytes, chorology, flora, Galicia, Spain.

Se aportan nuevos datos sobre briófitos recolectados recientemente en la localidad de Salvaterra de Miño (Pontevedra), de una importancia ecológica apreciable, considerados amenazados e incluidos en la Lista Roja de los Briófitos de la Península Ibérica (Sergio et al., 1994) con la categoría de especies raras y vulnerables respectivamente. La localidad de 